\title{
Lusioersily
}

\section{User centred design and validation during the development of domestic brain computer interface applications for people with acquired brain injury and therapists: a multi-stakeholder approach}

Daly-Lynn, J., Armstrong, E., \& Martin, S. (2016). User centred design and validation during the development of domestic brain computer interface applications for people with acquired brain injury and therapists: a multistakeholder approach. Journal of Assistive Technologies, 10(2), 67-78. https://doi.org/10.1108/JAT-01-20160002

Link to publication record in Ulster University Research Portal

Published in:

Journal of Assistive Technologies

Publication Status:

Published (in print/issue): 01/04/2016

DOI:

10.1108/JAT-01-2016-0002

Document Version

Author Accepted version

\section{General rights}

Copyright for the publications made accessible via Ulster University's Research Portal is retained by the author(s) and / or other copyright owners and it is a condition of accessing these publications that users recognise and abide by the legal requirements associated with these rights.

\section{Take down policy}

The Research Portal is Ulster University's institutional repository that provides access to Ulster's research outputs. Every effort has been made to ensure that content in the Research Portal does not infringe any person's rights, or applicable UK laws. If you discover content in the Research Portal that you believe breaches copyright or violates any law, please contact pure-support@ulster.ac.uk. 


\section{User centred design and validation during the development of domestic brain computer interface applications for people with acquired brain injury and therapists: a multi-stakeholder approach}

\section{Introduction}

Acquired brain injury (ABI) can have a profound impact on those who survive in terms of their physical and cognitive function. Medical advancements mean people are living for longer with more complex disabilities. There is no doubt that technology as assistive devices, which may or may not be digitally enabled, can support independence and autonomy. However, there is scope to harness the potential of new and emerging technologies and explore device integration and home based service availability. Currently available technologies do not always meet the needs of those who would benefit most from them, and often those with the most complex disabilities and associated needs by default are excluded from accessing services. To realise the potential of novel devices that are integrated into real world scenarios it is critical to get close to the voice and preferences of the people who will use these systems.

Acquiring and synthesising the requirements and preferences of people with complex disability requires thoughtful and skilled approaches by researchers. It is often necessary to develop alternative approaches to support people who currently fall into this gap, for example people living with locked in syndrome. This means a person has very limited ability to move, if any, and this can be an outcome of a severe ABI (Laurey et al., 2005). And yet, emerging technologies are the very devices and systems that have the potential to enable communication, independence and autonomy for the first time for this population (Tai et al., 2008).

Brain computer interfaces (BCI) offer a technology solution for people that have little or no ability to move their muscles to access digital systems and services (Daly and Wolpaw, 2008). These systems are controlled by brain waves harnessed through electrodes within a cap placed on the users head. BCI have shown promising potential as assistive devices for people living with complex disabilities to enable communication, autonomy and access to rehabilitation (Brunner et al., 2015). However, BCI still have a significant journey to go in terms of their design and development before they are a more robust and usable solution (Emiliani et al., 2011). Primarily, research has focused on individuals without a disability in a laboratory scenario with little exploration of migrating systems into the home environment and working directly with target end users. Therefore, a 'translational gap' emerged between end users requirements and the created systems of engineers. A shift towards the real world application of BCI, with target end user and stakeholders is essential to push these prototypes towards marketable assistive devices (Kubler et al., 2014). The overall focus of this research was to develop a BCI that could be used at home with the ambition of moving this technology to become an assistive device for daily use.

User centred design (UCD) is a multidisciplinary approach adopted to actively engage target users in the design and validation stages of device development to enhance usability (Mao et al., 2005). Gathering the needs, preferences and requirements of the target users is critical to inform each stage of the design process (Kujala, 2003). 
Research has indicated that engaging prospective consumers of assistive technologies at every level of design will ensure a comprehensive understanding of the needs for its proposed population, increasing end user acceptance, improving system quality, reducing device abandonment and aiding system usability (Kujala, 2003; Peterson, 2008; Van de Laar et al., 2011). In particular device abandonment in the provision of assistive technologies can be a significant issue (Cushman and Scherer, 1996). UCD offers a framework to support prioritising the needs of the users and in turn has the potential to reduce device abandonment by creating a better match between the technology and the user at an early stage of the design process. Additionally, the quality of the device can be increased by a clear focus on user requirements (Kujala, 2003).

The key principles, methods, theories and tools within UCD offer guidance to foster innovation. Damodaran (2001) describes the importance of incorporating 'an action research model' in order to consider the wide spanning impact of emerging technology on all the stakeholders. Action research offers a methodology to work with the multitude of stakeholders concerned with real world solutions to design systems to meet the needs of all the people who would use, set up, support and prescribe the assistive device. This could include people living with different conditions, caregivers, family, friends, support staff, healthcare workers and professionals that offer healthcare interventions such as occupational therapists and speech and language therapists. The framework offered by Damodaran (2001) includes a number of key components such as the active engagement of all the stakeholders, cycles of innovation and cycles of testing, and considering the technology in terms of the user and their environment. Also outlined by Basili and Turner (1975) this iterative enhancement increases the quality of systems and is a particularly useful tool for software development. The essential criteria is building the communication between the designers and the different type of users groups and stakeholders to enable innovation and share knowledge.

Theories are essential to shape the methodology incorporated into the UCD design process. Tools such as Jakob Nielsen's (1994) Ten Usability Characteristics, Shneiderman's (1992) Principles of Human Computer Interaction, and Bailey's (1982) Human Performance Model of Usability provide a reference for the technical developers to keep usability features at the centre of development. Furthermore, as we attempt to unpick the complexities of people, their ability levels, different living environments, roles and support systems, and how they use technology, holistic theoretical approaches are important to focus the evolution of the design process. PEO, which means Person, Environment \& Occupation (Law et al., 1996), is an example of one such approach that highlights the dynamic relationship between these human factors to support the developer's focus on maximising a person's ability through innovation.

Huggins et al., (2011) and Blain-Moraes et al., (2012) were among the first to engage with target end users to gather user requirements through surveys and focus groups in advance of the BCI design. The findings included the importance of accuracy, usability, aesthetic design, functionality, and reliability of the technology. Additionally, Kubler and her colleagues (2014) developed a UCD framework to evaluate BCI in accordance to the usability principles set out in ISO 9241-210. It was 
first introduced in 2011 to develop a standardised approach to incorporating target end users into all stages of the design life cycle (Zickler et al., 2011). It focuses specifically on the users experience operating the system in terms of its effectiveness, efficiency, and users satisfaction. Furthermore, this framework has already been incorporated into a number of studies successfully (Holz et al., 2015; Zickler et al., 2013).

This paper reports on the multi stakeholder engagement during the design, development and evaluation of an emerging assistive technology from one end user testing site within a larger European funded project called BackHome. It is beyond the scope of the paper to expand on the specific technology developed, or the work of other partners however this work will be referenced to enable interested readers to investigate different aspects of this work in greater depth. The aim is to outline the vital role of all those parties who will use, support the use, and prescribe the use of this type of assistive device in the future when it is a marketable product with the ability to support independence, autonomy and choice for those living with ABI.

\section{Methodology}

The multi-stakeholder approach incorporates both qualitative and quantitative methods of data collection. The system at the centre of development was a BCI with a wide range of applications added at different stages of development including a speller for communication, web access, Brainpainting (computer based painting application), cognitive rehabilitation (find a category and card sorting memory task recommended by therapists), and a multimedia player (Mirelles et al., 2015a). A therapist station was also developed concurrently to include a number of functionalities to support home use such as to access the users satisfaction with the $\mathrm{BCI}$ remotely. It also enabled the user to schedule cognitive rehabilitation tasks and receive the results of the tasks and the quality of life questionnaires (Mirelles et al., 2015a). A cap with encased electrodes to harness the EEG from participants was developed throughout this innovative design cycle and is described in Hintermuller et al., (2015). An ethical framework was set out to ensure best practice was incorporated into the recruitment of participants and engagement with all stakeholders throughout the lifecycle of the project (Daly et al., 2015a). The University of Ulster Ethics Committee and Office for Research Ethics Northern Ireland (ORECNI) provided ethical approval for the different stages of engagement with the range of stakeholders.

\section{Stakeholder Recruitment}

Three key stakeholder groups were identified as participants for this study: target end users post ABI, caregivers and professionals described as therapists such as occupational therapists, physiotherapists, and speech and language therapists providing healthcare interventions for people with $\mathrm{ABI}$ in the community. All end user participants had a diagnosis of an ABI, were over eighteen years of age, post rehabilitation, living in the community, and no longer receiving acute sector services. In phases two and three of the design cycle participants with a history of epilepsy were screened out because of the flashing lights of the P300 BCI. It must be noted there is no evidence of BCI stimulus inducing a seizure however as a safeguarding precaution it was decided as best practice to adopt this approach. Caregivers were 
individuals that voluntarily provided care for a person after an ABI such as a family member or a friend. Therapists were primarily occupational therapists, and speech and language therapists working with clients in the community after an ABI.

\section{Stakeholder Engagement}

The interactive design cycle is illustrated in Figure 1 to outline the three clear stages within this methodological framework. Each stage is clearly defined in Table 1 in terms of the procedure adopted and the participants that took part at that stage.

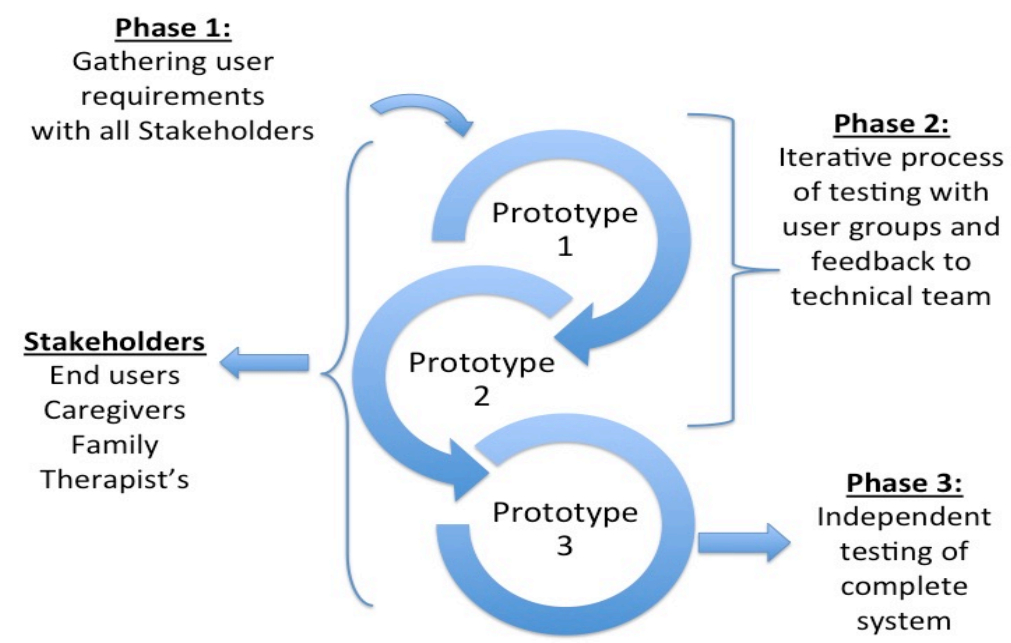

Figure 1: Iterative design life cycle

\section{Phase 1: Gathering user requirements}

Each focus group commenced with a PowerPoint introduction to the project and BCI systems. Participants were asked to consider the benefits and barriers of BCI design, potential BCI applications and the use of BCI technology in everyday life. The recordings from the groups were transcribed and analysed using thematic analysis.

\section{$>$ Table $1<$}

\section{Phase two: Iterative Design Approach}

The BCI system used a P300 paradigm and had two monitors as illustrated in Figure 2, the P300 interface and the monitor for applications to be displayed. The hardware will remain the same for prototype one and two but the software will be advanced according to the outcome of this iterative stage. The testing during phase two moved the BCI out of the laboratory into a rehabilitation center with real end users and a control group set up by researchers naive to BCI. The testing phase required each participant to complete a set protocol on three occasions each as set out in Table 1 . During the protocol if the participant made three unsuccessful attempts to select a symbol at one step they were directed to the next step in the protocol and erroneous selections were not corrected. The user satisfaction questionnaires contained the extended QUEST 2.0 (Zickler et al 2011) with added items specific to BCI: Reliability, Speed, Learnability and Aesthetic Design. Additionally, the VAS scale asked users to rate their satisfaction with the device each time they used it from 0 (not satisfied) to 10 (very satisfied) and the NASA TLX is a commonly used standardised tool to measure perceived workload of users. 
Phase Three: Independent Testing of Final System

The final phase of the design process involved evaluating two components of the system: the final BCI prototype and therapist station (Figure 2). These systems intertwined within the evaluation however the key stakeholders each had separate roles.
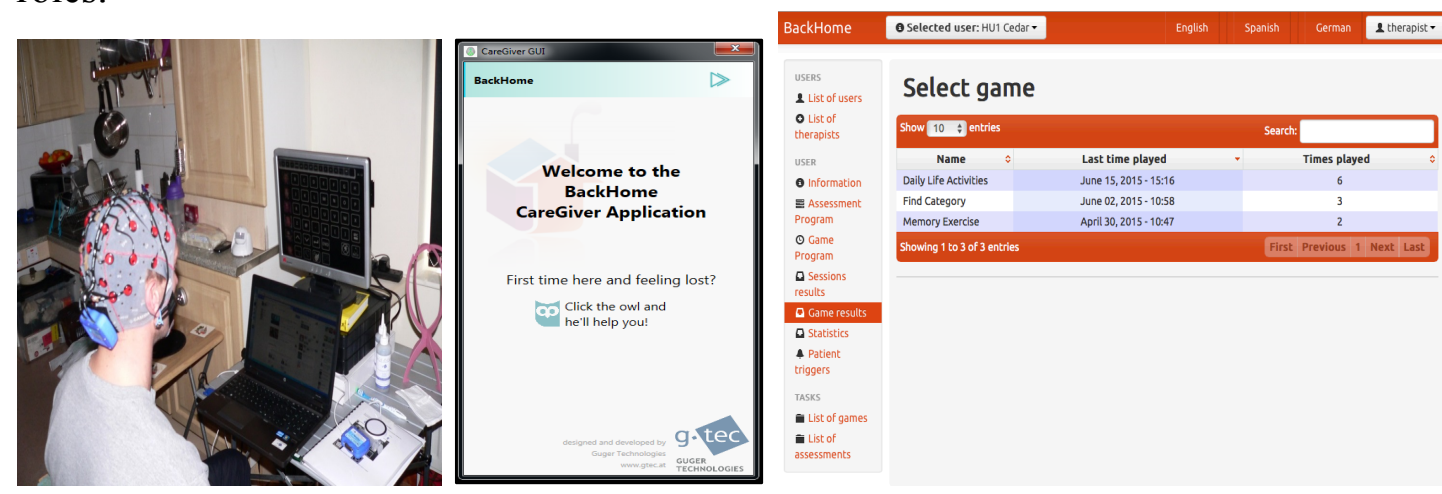

Figure 2: a) Home based BCI; b) Caregiver Interface; c) therapist station

Over the six weeks, target end user participants were asked to complete a specified task from one of the available applications, a cognitive rehabilitation task, and one other task of their choosing each week. Cognitive rehabilitation tasks were scheduled through the therapist's station and the satisfaction with BCI performance VAS and perceived rate of control was also assessed through this platform. At the end of the six-week period the participants were asked to complete the usability questionnaires. During the six-week evaluation caregivers were required to set the user up on the system each time. Therapists were invited to focus groups on the final BCI system and therapist station. Additionally, they were given the opportunity to work through a protocol on the therapist station independently and complete a final questionnaire on its functionality.

\section{Results}

\section{1) Gathering User Requirements}

The four focus groups with end users (EU) and their caregivers generated wide spanning conversations about the technology from the hardware, to $\mathrm{BCI}$ as an enabling technology, to its use in the acute stages post ABI. Interestingly, the BCI was perceived to give back a sense of achievement, take away boredom, improve quality of life, and motivate the user to undertake rehabilitation. The potential for the $\mathrm{BCI}$ to follow the recovery from hospital to home in order to increase the rehabilitation process was also outlined. The usability of the BCI for both the end user and the caregiver was a key priority within the groups 'hat on, reboot and out the door' (EU 14)

It was also important that the BCI could facilitate a person to carry out as many tasks as possible including call their caregiver 'It could send a pre recorded message saying that I need assistance' (EU 17). Suggestions for the functionality of the BCI included communication, surfing the Internet, gaming, operating wheelchair, driving a car, incorporating a spelling assistance, using a mobile phone and smart TV. 'Through their smart TV they can have a working life and that answer's your question of how to 
get rid of boredom' (EU 13). Here is a summary of an outcome from the four focus groups:

- Appearance and functionality of the cap is an important factor to consider

- Dry or non-gel based electrodes would be welcome

- Simple, reliable, user-friendly technology is important for the non-expert

- Offer a range of applications for use in everyday life

- The ability to personalise the functionality of the system is important

- Impact on fatigue level will correlate to utility of the BCI system

- Safety and privacy issues should be considered

$>$ Table $2<$

The discussion with therapists explored their views of BCI as an assistive technology. Summaries of the challenges they envisage are outlined in Table 2. In addition to the discussion therapists were asked some set questions and the responses are illustrated in Table 3.

$>$ Table $3:<$

\section{2) Iterative Design and Evaluation}

This design phase focused on the evaluation of the BCI prototype in an iterative process to improve the design features and maximise its functionality for home based testing. The second prototype testing included the same sample as prototype one except for one end user that withdrew from the testing. The participant withdrew because the system was not responding sufficiently for him.

$>$ Table $4<$

The outcome from the testing of prototype one was fed back to the technical developers to implement improvements for the second iterative of the prototype. The technical improvements included increasing the functionality from Facebook, Twitter and environmental control to all of the available options; alter the P300 stimulus to Famous Faces (Kaufmann et al., 2011) to enhance the users experience interacting with the system; and increase the stability of the system. Table 4 gives a summary of the outcome from the testing for both prototype one and prototype two with a control group and end users living with ABI (Daly et al., 2015a; Daly et al., 2015b). The overall accuracy score for end users greatly increased from prototype one (55\% $( \pm 10.6))$ compared to prototype two $(76 \%( \pm 11.5))$. The increase in the accuracy scores in the second iteration, was attributed to the P300 paradigm with new software 'Famous Faces' stimulus, which is considered to enhance eye focus (Kaufmann et al., 2011). Feedback from the testing is set out in Table 5 below to illustrate the progress of the system according to the outcome from each stage of testing and the improvements made to the final prototype. 


\section{Therapists Engagement in Iterative Design}

Through this phase of iterative design, development and testing a group of

Occupational therapists and Speech and Language therapists were recruited to work in focus groups to develop the cognitive rehabilitation tasks for the BCI and provide feedback on the therapist station. The three cognitive rehabilitation tasks were developed by the technical design team and refined by therapists over three sessions $(\mathrm{N}=10 ; \mathrm{N}=9 ; \mathrm{N}=3)$ according to their expertise providing rehabilitation for clients post ABI. Finally the tasks were included on prototype two where feasibility testing was undertaken. Feedback from this testing was given to the technical team and a final version of the cognitive rehabilitation tasks were available on the final system.

$>$ Table $5:<$

\section{3) Final System Evaluation}

The iterative design approach moved both the BCI and therapist station into a final evaluation stage with the newest innovations developed within the project such as the wireless cap and an easy to use set up for non expert caregivers (Mirelles et al., 2015b). New lessons were learnt when these complex systems move out of the laboratory for the first time and these are outlined in the discussion. Overall, participants were enthusiastic about the $\mathrm{BCI}$ and their experience evaluating it over the six weeks period at home (See Table 6). Additionally, non-expert caregivers were able to support the user by setting them up on the prototype and at times help participants to navigate through the system. Key feedback from the caregivers included the set up needs to be even easier, the BCI software needs to be more stable, users need to be able to use the system completely independently after set up, and the time required for the set up needs to be shorter. Interestingly the technical problems that emerged for caregivers during the set up were primarily due to signal interference within the home environment that had not been identified in previous tests in controlled environments.

$>$ Table $6<$

The findings from the therapist's focus groups and usability questionnaire after testing the functionality of the platform are outlined in Table 6. Some qualitative statements from therapists included: 'Allows you to easily access patient results'; 'Easy to set up tasks for patient to complete'; 'very useful starting point when client returns home from hospital and is very dependant'; It is difficult to increase and develop complexity of task'; 'more variety of tasks would have been beneficial'.

\section{Discussion}


The UCD outlined has incorporated multi-stakeholder engagement at three stages of iterative design to develop the BackHome system for home use. A number of challenges emerged when adopting this framework such as the time needed to incorporate these methods into the design process, the ability to access and keep this target sample engaged and the translation of information from the technical developers to end users. Table 6 sets out the specific challenges experienced at each stage of the design phase for the sake of clarity. These challenges are useful lessons in the development of any type of assistive device incorporating UCD methodology. A significant challenge in UCD is access to the sample (Kubler et al., 2014). The BackHome consortium brought together academia, industry and end user partners, which enabled the communication between the technical developers and the stakeholders. A multidisciplinary approach is useful to streamline the access to end users through a partner providing services for people living with ABI, however, this also meant there were no technical experts onsite to resolve problems that emerged with the unstable prototypes.

\section{$>$ Table $7<$}

Keeping participants engaged throughout the lifecycle of the methodology was particularly challenging. The variation of performance and the effort required for users to interact with the BCI when it was unreliable meant that participants were not very motivated to stay engaged in testing by the end of prototype two and this was also apparent during home use when the system was not responding sufficiently. In line with previous research, BCI reliability and accuracy were key factors to user engagement (Kubler et al., 2013). Due to time constraints and workload pressure for healthcare professionals the numbers engaged in the design of cognitive rehabilitation tasks reduced considerable by the last working group. Additionally, time can be a specific barrier for caregivers to contribute to UCD due to the number of roles they facilitate on a daily bases particularly in terms of the time required to set up the system three times a week for the home testing. Ensuring that participant experience is meaningful is also essential during UCD. Interestingly, end users and therapists were satisfied to see their feedback implemented into future interactions of the prototypes.

A wide range of novel innovations, inline with the findings of the stakeholder focus groups and the iterative testing, were implemented into the final home-based prototype (see Table 5). This included a wireless electrode cap to improve appearance, the option to use the dry electrodes, an algorithm called dynamic stopping method to reduce the effort needed by users to control the BCI and moving onto a different software platform to make the system more reliable. However, the wireless cap was sometimes challenging for the caregiver to connect to the base station, the dry electrodes could not establish a signal good enough to be used at home, users still required significant effort to operate the $\mathrm{BCI}$ and the system would still crash from time to time. Therefore, the aspirations to meet all the needs of the focus groups were not realised. However, the system was safe for users to operate, it offered a wide range of applications including the ability to progress with cognitive rehabilitation and it was easy enough for a non-expert to set. System integration was multidimensional with aspects of the system becoming unreliable prior to final 
validation. The transition from lab to home often destabilised the system, which required technical expertise at the home site for initial set up. A home set up and stabilisation phase would have been a useful additional stage to stabilise the set up and minimise challenges that emerged during this final evaluation.

Ethical issues and safeguarding all participants was central to the user centred methodology adopted. An ethical framework was developed to ensure that end users were approached to participate in accordance to best practice and strategies were put in place in order to appropriately deal with any unforeseen events. Users did not report experiencing residual effects from interacting with the BCI apart from feelings of fatigue in some incidents. A first aid officer was informed in advance of each testing session to ensure immediate action could be taken in the event a participant was starting to feel unwell, but this never occurred. Obtaining ethical approval is a significant challenge within a UCD framework due to the very fact that the technology is in the design and evolution phase (Carmichael and Carmichael, 2013). In addition the phased approach and emergence of technology over time in the project necessitated numerous amendments to ethical approval, such as notifications on the iterative changes made to the prototype by the developers and moving the testing into a domestic environment.

BCI research is only beginning its journey from the laboratory into the end user's home environment. This is an important time for this research and to work directly with the many people required to use, set up and deliver services is critical. User centred design is essential as a methodological framework to ensure the appropriate development of emerging technologies with all the stakeholders involved. As outlined above wide ranging challenges emerged during the project however with thoughtful and sensitive handling most could be overcome or worked around. These challenges are useful lessons for research teams incorporating UCD in the development of any type of assistive technology and enable teams to implement strategies to mitigate risk in the development of proposals, projects and in applications for funding. More research is needed to bring these technologies to the market place to enable real world application for people with complex disability, to enable caregivers to set users up on these systems as part of their daily routine and to be adopted to support therapeutic practice. This research outlines the potential of BCI as an assistive technology and the process of incorporating UCD as a theoretical framework in its development.

\section{Acknowledgement}

The research leading to these results has received funding from the European Community's, Seventh Framework Programme FP7/2007-2013, BackHome project grant agreement $\mathrm{n}^{\circ} 288566$ ". We would like to thank all the project partners that contributed to each phase of this work. 


\section{References}

Bailey, R.W. (1982) Human performance engineering: A guide for system designers. Prentice Hall Professional Technical Reference.

Basili, V. R., \& Turner, A. J. (1975) 'Iterative enhancement: A practical technique for software development.' IEEE Transactions on Software Engineering, SE-1(4), pp 390-396. doi:10.1109/TSE.1975.6312870

Blain-Moraes, S., Schaff, R., Gruis, K.L., Huggins, J.E. and Wren, P.A. (2012) 'Barriers to and mediators of brain-computer interface user acceptance: focus group findings', Ergonomics, 55(5), pp. 516-525.

Brunner, C., Birbaumer, N., Blankertz, B., Guger, C., Kübler, A., Mattia, D., Millán, J.d.R., Miralles, F., Nijholt, A. and Opisso, E. (2015) 'BNCI Horizon 2020: towards a roadmap for the BCI community', Brain-Computer Interfaces, (ahead-of-print), pp. 110 .

Carmichael, C. and Carmichael, P. (2013) 'BNCI systems as a potential assistive technology: ethical issues and participatory research in the BrainAble project', Disability and Rehabilitation: Assistive Technology, 9(1), pp. 41-47.

Cushman, L., \& Scherer, M. (1996) 'Measuring the relationship of assistive technology use, functional status over time, and consumer-therapist perceptions of ATs'. Assistive Technology, 8(2), pp.103-109.

Daly, J.J. and Wolpaw, J.R. (2008) 'Brain-computer interfaces in neurological rehabilitation', The Lancet Neurology, 7(11), pp. 1032-1043.

Daly, J., Armstrong, E., Pinegger, A., Müller-Putz, G. and Martin, S. (2015a) 'In pursuit of an easy to use brain computer interface for domestic use in a population with brain injury', International journal on recent and innovation trends in computing and communication, 3(6), pp. 4019-4029.

Daly, J., Armstrong, E., Thomson, E., Andreas, P. and Martin, S. (2015b) 'P300 brain computer interface control after an acquired brain injury.', International Journal on recent and innovation trends in computing and communication, 3(1), pp. 318-325.

Damodaran, L. (2001) 'Human factors in the digital world enhancing life style - the challenge for emerging technologies', International Journal of Human-Computer Studies, 55(4), pp. 377-403.

Demers, L., Weiss-Lambrou, R. and Ska, B. (2002) 'The Quebec User Evaluation of Satisfaction with Assistive Technology (QUEST 2.0): an overview and recent progress', Technology and Disability, 14(3), pp. 101-105.

Emiliani, P.L., Stephanidis, C. and Vanderheiden, G. (2011) 'Technology and inclusion-Past, present and foreseeable future', Technology and Disability, 23(3), pp. 101. 
Hart, S.G. and Staveland, L.E. (1988) 'Development of NASA-TLX (Task Load Index): Results of empirical and theoretical research', Advances in Psychology, 52, pp. 139-183.

Hintermüller, C., Vargiu, E., Halder, S., Daly, J., Miralles, F., Lowish, H., Anderson, N., Martin, S. and Edlinger, G. (2015) 'Brain Neural Computer Interface for Everyday Home Usage' In Universal Access in Human-Computer Interaction. Access to Interaction Springer, pp. 437-446.

Holz, E.M., Botrel, L., Kaufmann, T. and Kübler, A. (2015) 'Long-term independent brain-computer interface home use improves quality of life of a patient in the lockedin state: a case study', Archives of Physical Medicine and Rehabilitation, 96(3), pp. S16-S26.

ISO, C. (2008) '9241-210: Ergonomics of human-system interaction-Part 210: Human-centred design process for interactive systems', International Organization for Standardization, Geneva.

Kaufmann, T., Schulz, S., Grünzinger, C. and Kübler, A. (2011) 'Flashing characters with famous faces improves ERP-based brain-computer interface performance', Journal of neural engineering, 8(5), pp. 056016.

Kübler, A., Holz, E.M., Riccio, A., Zickler, C., Kaufmann, T., Kleih, S.C., StaigerSälzer, P., Desideri, L., Hoogerwerf, E. and Mattia, D. (2014) 'The User-Centered Design as novel perspective for evaluating the usability of BCI-controlled applications', PloS one, 9(12), pp. e112392.

Kübler, A., Holz, E., Kaufmann, T. and Zickler, C., 2013. A user centred approach for bringing BCI controlled applications to end-users. Brain-Computer Interface SystemsRecent Progress and Future Prospects.

Kujala, S. (2003) 'User involvement: a review of the benefits and challenges', Behaviour \& information technology, 22(1), pp. 1-16.

Laar, B., Nijboer, F., Gürkök, H., Plass-Oude Bos, D. and Nijholt, A. (2011) 'User experience evaluation in BCI: Bridge the gap', International Journal of Bioelectromagnetism, 13(3), pp. 157-158.

Laureys, S., Pellas, F., Van Eeckhout, P., Ghorbel, S., Schnakers, C., Perrin, F., Berre, J., Faymonville, M., Pantke, K. and Damas, F. (2005) 'The locked-in syndrome: what is it like to be conscious but paralyzed and voiceless?', Progress in brain research, 150, pp. 495-611.

Law, M., Cooper, B., Strong, S., Stewart, D., Rigby, P. and Letts, L. (1996) 'The person-environment-occupation model: A transactive approach to occupational performance', Canadian Journal of Occupational Therapy, 63(1), pp. 9-23.

Mao, J., Vredenburg, K., Smith, P.W. and Carey, T. (2005) 'The state of user-centered design practice', Communications of the ACM, 48(3), pp. 105-109. 
Miralles, F., Vargiu, E., Dauwalder, S., Solà, M., Müller-Putz, G., Wriessnegger, S.C., Pinegger, A., Kübler, A., Halder, S., Käthner, I. et al. (2015a) 'Brain Computer Interface on Track to Home', The Scientific World Journal - Article ID 623896.

Miralles, F., Vargiu, E., Rafael-Palou, X., Solà, M., Dauwalder, S., Guger, C., Hintermüller, C., Espinosa, A., Lowish, H., Martin, S., Armstrong, E., and Daly, J. (2015b) 'Brain Computer Interfaces on Track to Home: Results of the Evaluation at Disabled End-Users's Homes and Lessons Learnt', Frontiers in ICT, 2, pp. 25.

Nielsen, J. (1994) Usability engineering. Elsevier.

Peterson, W. (2008) 'Role of persons with disability in the design process', Topics in Stroke Rehabilitation, 15(2), pp. 87-96.

Shneiderman, B. (1992) Designing the user interface: strategies for effective humancomputer interaction. Addison-Wesley Reading, MA.

Tai, K., Blain, S. and Chau, T. (2008) 'A review of emerging access technologies for individuals with severe motor impairments', Assistive Technology, 20(4), pp. 204-221.

Tamburrini, G. (2009) 'Brain to computer communication: ethical perspectives on interaction models', Neuroethics, 2(3), pp. 137-149.

Zickler, C., Halder, S., Kleih, S.C., Herbert, C. and Kübler, A. (2013) 'Brain Painting: Usability testing according to the user-centered design in end users with severe motor paralysis', Artificial Intelligence in Medicine, 59(2), pp. 99-110.

Zickler, C., Riccio, A., Leotta, F., Hillian-Tress, S., Halder, S., Holz, E., StaigerSalzer, P., Hoogerwerf, E.J., Desideri, L., Mattia, D. and Kubler, A. (2011) 'A braincomputer interface as input channel for a standard assistive technology software', Clinical EEG and neuroscience, 42(4), pp. 236-244. 
PEER REVIEWED PAPER 